\title{
Monte Carlo Studies for the Radiation Shielding Design of LCLS-II
}

\author{
Shanjie Xiao*, Stan X. Mao, Ludovic Nicolas, Sayed H. Rokni, Mario Santana Leitner \\ SLAC National Accelerator Laboratory, 2575 Sand Hill Road, Menlo Park, California, 94025, USA
}

\begin{abstract}
Intensive Monte Carlo simulations performed with state-of-the-art computation codes are applied to the radiation shielding design of LCLS-II, which will be the extension of Linac Coherent Light Source (LCLS) at SLAC and will use the middle one-third of SLAC two-mile Linac. This paper describes the Monte Carlo studies of the first and last system where electron beams are involved, namely the LCLS-II Injector and the X-ray Transport and Diagnostics System (XTOD).
\end{abstract}

Keywords: Monte Carlo; radiation shielding; free electron laser; LCLS; FLUKA

\section{Introduction}

LCLS-II will be the extension of Linac Coherent Light Source (LCLS), which is the first hard X-ray free electron laser facility, and will use the middle one-third of SLAC two-mile Linac. Figure 1 shows the overall scheme of the radiation safety system of LCLS-II [1].

This paper describes Monte Carlo studies of the first and last systems where electron beams are involved, namely the LCLS-II Injector and the X-ray Transport and Diagnostics System (XTOD).

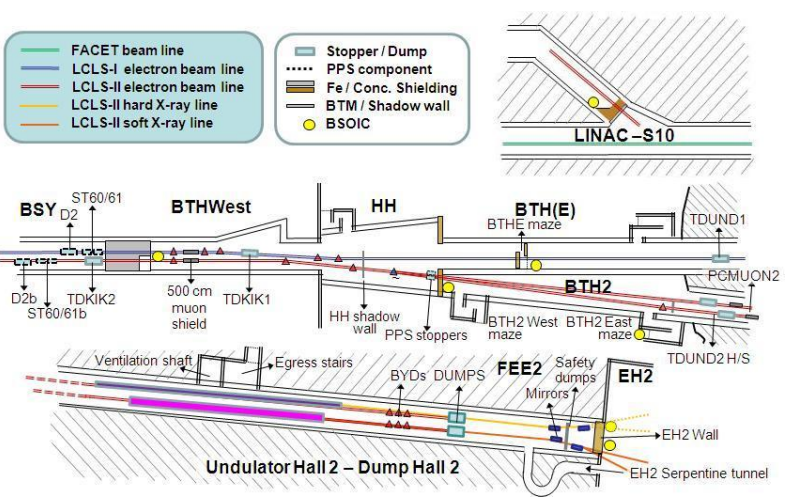

Figure 1. Overall scheme of LCLS-II radiation safety system

\section{Monte Carlo Studies for LCLS-II Radiation Shielding}

\subsection{LCLS-II injector}

\subsubsection{Sector-10 injector wall}

The injector will be the first construction of LCLS-II. It will be built in the Sector-10 vault, contemporarily to the operation of FACET (Facility for Advanced Accelerator Experimental Tests) at the Sector-10 in the

*Corresponding author. Email: xiaosj@slac.stanford.edu
Linac. Therefore, a shielding wall separating the S10 vault and Linac area has been designed, with the help of comprehensive FLUKA [2,3] simulations, to protect personnel in LCLS-II injector construction. FACET has a chicane with two legs to compress electron and position bunches beside the injector vault. The wall design is based on the FACET beam loss scenarios and the corresponding dose limitations listed in Table 1. The dose limitation corresponds to normal $10 \mathrm{~W}$ losses when chicane is "ON" is the most restricted one and thus defines the wall design. This paper focuses on radiation doses under normal loss scenarios.

Table 1. FACET beam loss scenarios and shield criteria.

\begin{tabular}{llll}
\hline \multicolumn{1}{c}{ Scenario } & \multicolumn{1}{c}{ Beam Loss } & \multicolumn{1}{c}{ Dose Limitation } \\
\hline \multirow{4}{*}{ Normal } & Chicane & $10 \mathrm{GeV}, 10 \mathrm{~W}$ & $10 \mathrm{mSv} /$ year \\
& ON & (5 W on each leg) & $\begin{array}{l}(5 \mu \mathrm{Sv} / \mathrm{h} \text { for } 2000 \\
\text { hours })\end{array}$ \\
\cline { 2 - 3 } & Chicane & $10 \mathrm{GeV}, 5 \mathrm{~W}$ on & \\
OFF & straight line & $0.5 \mu \mathrm{Sv} / \mathrm{h} / \mathrm{W}$ \\
\hline Mis-steering & $10 \mathrm{GeV}, 10 \mathrm{~W}$ & $4 \mathrm{mSv} / \mathrm{h}$ \\
& & $0.4 \mathrm{mSv} / \mathrm{h} / \mathrm{W}$ \\
\hline $\begin{array}{l}\text { Maximum credible } \\
\text { beam }\end{array}$ & $14 \mathrm{GeV}, 62 \mathrm{~kW}$ & $0.25 \mathrm{~Sv} / \mathrm{h}$ \\
\hline
\end{tabular}

Figure 2 shows the top view of the shielding wall with FACET chicane magnets and a 3D representation of the wall. The wall consists of poured concrete and steel embedded in concrete to fit into the limited space defined by the injector design. Since beam losses may happen at any location along the chicane, seven (7) representative loss points were selected for simulations. Figure 3 shows the radiation dose (using FLAIR [4]) at beam height behind the designed S10 wall when $10 \mathrm{~W}$ beam is lost on the straight section between chicane magnets, where beam losses can deliver the largest dose to the injector vault. Note that the dose is in the linear scale of $1-10 \mu \mathrm{Sv} / \mathrm{h}$ and the dimension is in the unit of 
centimeter. Other than the worst case, dose behind the wall is less than $5 \mu \mathrm{Sv} / \mathrm{h}$. Because beam loss will not be on a fixed position, the overall doses behind the wall meet the design criteria. Also passive dosimeters (e.g. TLDs) will be used inside the injector vault to monitor the long-term radiation dose.
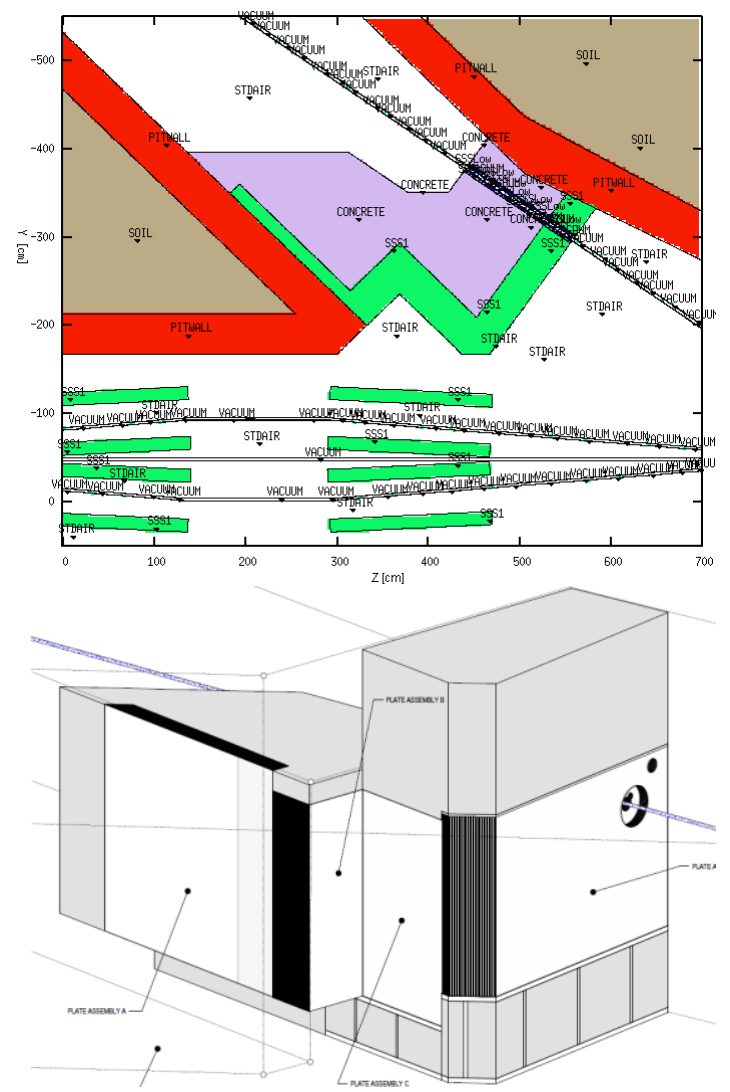

Figure 2. Design of S10 wall: top view and 3D scheme.

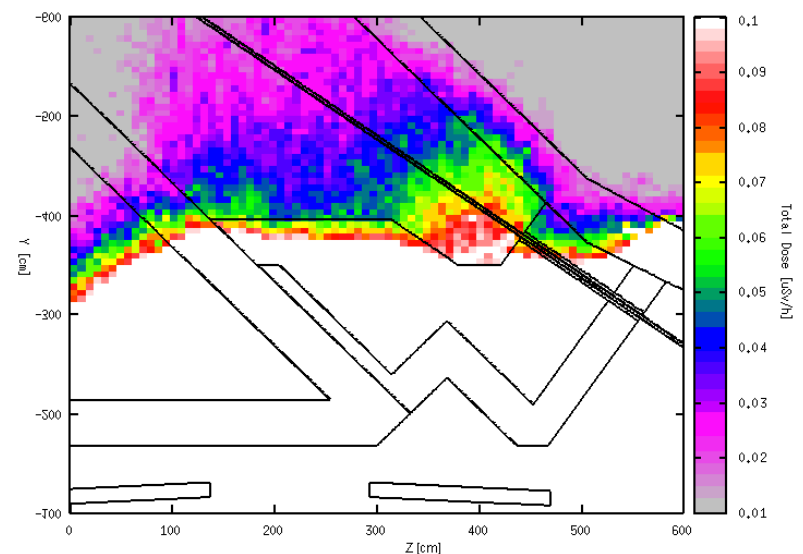

Figure 3. Total dose at beam height behind S10 wall in the worst scenario.

\subsubsection{Shielding for injector operation}

During injector operations, electrons are accelerated in the injector vault and sent to a dump inside the Linac tunnel. There are three penetrations from the injector vault to the laser room on ground: one for the injector gun laser, one for air ventilation, and a stairway for personnel access. During injector operation, the prompt radiation due to beam loss on injector components will propagate through these penetrations.

FLUKA simulations were performed to study doses through penetrations for beam losses from various possible loss points. Figure 4 shows the worst case for the stairway opening, when $25 \mathrm{~W} 64 \mathrm{MeV}$ electrons are lost on a Faraday cup after the first accelerator section. The total dose at the exit plane is about $30 \mu \mathrm{Sv} / \mathrm{h}$. But the top of the stairway is not accessible during injector operation. The PPS (personal protection system) barrier will limit the accessible area on ground. If the barrier is $30 \mathrm{~cm}$ away from the stairway opening, the total dose will be less than $5 \mu \mathrm{Sv} / \mathrm{h}$.

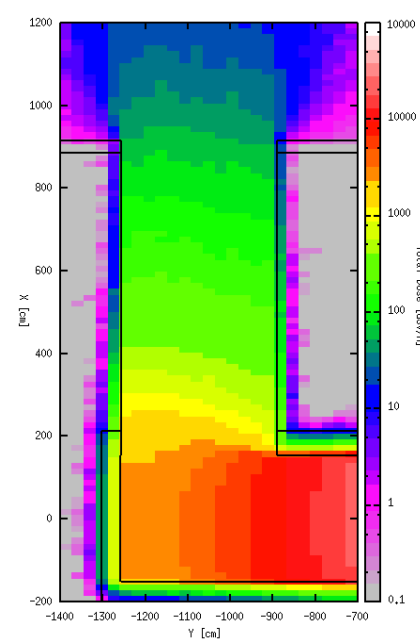

Figure 4. Vertical view of total dose for the stairway opening in the worst scenario.

\subsubsection{Residual activities from injector dump}

The LCLS-II injector will be operated in two phases: the injector will be used for R\&D (Phase-I, deliver the beam to a temporary dump) in the 1-2 years prior to it being used as the LCLS-II linac injector (Phase-II, deliver the beam either to the spectrometer branch line or to the main Linac). $50 \mathrm{~W}$ electron beams can be sent to the spectrometer line and terminated by a copper dump. The use factor of this dump is small from the experience of LCLS-I. It is assumed that the dump is irradiated continuously for one day in simulations.

Side shielding is required to reduce residual dose in the walking aisle to less than $50 \mu \mathrm{Sv} / \mathrm{h}$. Figure 5 shows residual doses after 1 hour cooling from the shielding of different materials. The side shielding is either 6" $(\mathrm{a} \& \mathrm{~b})$ or 3" (c\&d) beside dump and thinner in the upstream, 4" $(a \& b)$ or 2" (c\&d). It is noticeable that although the side shielding made of A36 steel and stainless steel (a\&b) has double thickness of shielding made of cold roll steel and natural iron (c\&d), the residual with the former (40-60 $\mu \mathrm{Sv} / \mathrm{h})$ are still higher than dose with the latter (30-40 $\mu \mathrm{Sv} / \mathrm{h}$ ). Manganese and copper inside A36 steel and stainless steel is activated by secondary radiation from the dump and contribute noticeable residual dose. Therefore, the residual dose shielding should be made from cold roll steel or natural iron or other steel with a limited fraction of manganese and copper. 


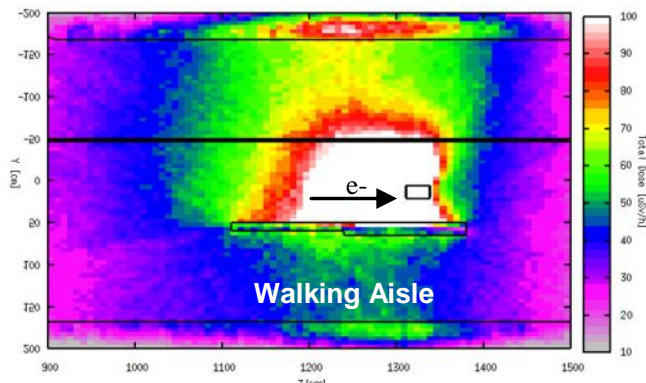

(a) $\mathrm{A} 36(4 "+6 ")$

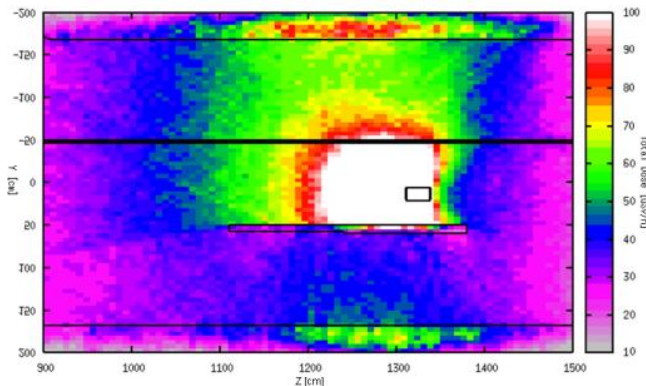

(c) Cold Roll Steel (2" +3 ")

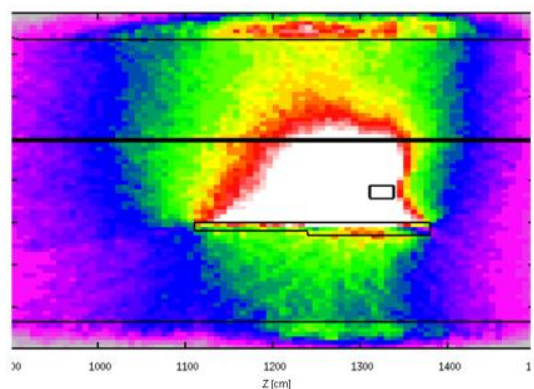

(b) Stainless Steel (4" + 6")

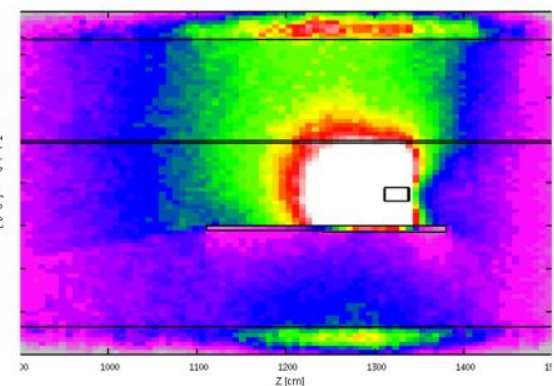

(d) Natural Iron (2" + 3")

Figure 5. Residual dose $[\mu \mathrm{Sv} / \mathrm{h}]$ from dump after 1 day irrandiation and 1 hour cooling with the shielding of different materials.

\subsection{X-ray Transport and Diagnostics System (XTOD)}

The XTOD region combines the undulator hall downstream of the main electron dump and the front end enclosure (FEE). There is no wall between the undulator hall and FEE in LCLS-II design. An end wall (called EH2 wall) will separate XTOD and the experimental hall EH2. The XTOD system includes a set of fixed and insertable mirrors, collimators, diagnostics, and other $\mathrm{x}$-ray instruments needed to characterize $\mathrm{x}$-ray beams and transport them to the experimental hall or to a photon beam stopper. The end wall protects personnel in the new experimental hall from radiation generated in LCLS-II tunnel, and the mirror and collimator system can filter out undesirable radiation to the level allowing personnel access around beam pipes in the experimental hall. Since XTOD systems are still in active evolution, this paper uses the SXR (soft X-ray beamline) only and focuses on principles, while not on final design. Figure $\mathbf{6}$ shows the latest layout of SXR, which consists of three mirrors with the $2^{\text {nd }}$ mirror being insertable.

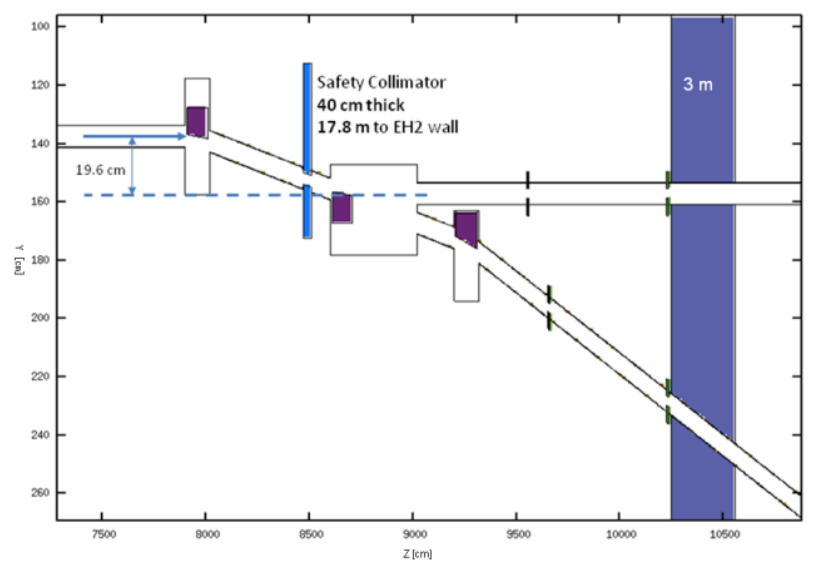

Figure 6. Scheme layout of XTOD SXR.

\subsubsection{Radiation shower to experimental hall}

Electrons generate bremsstrahlung radiation through collisions with residual gas molecules, certain portion of electron beams is lost on the bending dipoles sending electrons to the main dump, and from time to time, beam intercepts objects such as wire scanners or screens that are inserted into the beam. Analysis [5] shows that 200 $\mathrm{mW}$ bremsstrahlung from normal $5 \mathrm{~kW} 15 \mathrm{GeV}$ electron beams is a conservative estimation, and the dose behind $\mathrm{EH} 2$ wall should be less than $0.5 \mu \mathrm{Sv} / \mathrm{h}$. In accidents, electrons may also be sent to XTOD, and the dose rate behind EH2 wall should be less than $250 \mathrm{mSv} / \mathrm{h}$ under the maximum credible beam (MCB) of $10 \mathrm{~kW} 15 \mathrm{GeV}$ electrons [5].

It is found that a local safety collimator after the first mirror can help reduce the thickness and cost of the end wall. Figure 7 shows the total dose with a $40 \mathrm{~cm}$ iron safety collimator and $3 \mathrm{~m}$ concrete EH2 wall. The highest dose is on the primary incident direction. The dose behind the wall is $0.21 \mu \mathrm{Sv} / \mathrm{h}$, of which $60 \%$ is from muons, $20 \%$ from photons and $20 \%$ from neutrons. For comparison, LCLS-I applied two walls with a total of $2.1 \mathrm{~m}$ iron and $1.8 \mathrm{~m}$ concrete. The dose in accident with this design is $8.3 \mathrm{mSv} / \mathrm{h}$, under limitation also.

\subsubsection{Radiation leakage from beam pipe}

On the other hand, bremsstrahlung, spontaneous $\mathrm{x}$-rays and the desired free-electron laser (FEL) X-rays will interact with XTOD mirror sets, and certain amount of radiation will enter the experimental hall from beam pipes. Access around beam pipe during operation is expected, therefore the XTOD mirror and collimator system should limit the radiation leakage so as to reduce the dose rate at $30 \mathrm{~cm}$ away from beam pipes in the experimental hall to less than $0.5 \mu \mathrm{Sv} / \mathrm{h}$.

The SXR configuration shown in Figure 6 has two collimators between the last mirror and $\mathrm{EH} 2$ wall on 
each branch line, and each mirror reflects x-rays 28 mrad. Each collimator is $8 \mathrm{~cm}$ long heavy tungsten alloy with the inner diameter of $33 \mathrm{~mm}$ and outer diameter of $15 \mathrm{~cm}$. The trace of radiation leakage in $\mathrm{EH} 2$ can be found in Figure 7, but for more accurate estimation a two-step simulation was performed: (1) record all particles (location, direction, energy and weight) reaching $\mathrm{EH} 2$ from beam pipe into a file; (2) use the recorded particles to simulate radiation dose inside $\mathrm{EH} 2$. Figure 8 shows the total dose when the leaked radiation interacts with a thin target $(1 \mathrm{~cm}$ iron) inside the beam pipe. The total dose is about $1 \mu \mathrm{Sv} / \mathrm{h}$ on contact with the beam pipe and $0.1 \mu \mathrm{Sv} / \mathrm{h}$ at $30 \mathrm{~cm}$ away, well below the requirement of $0.5 \mu \mathrm{Sv} / \mathrm{h}$.
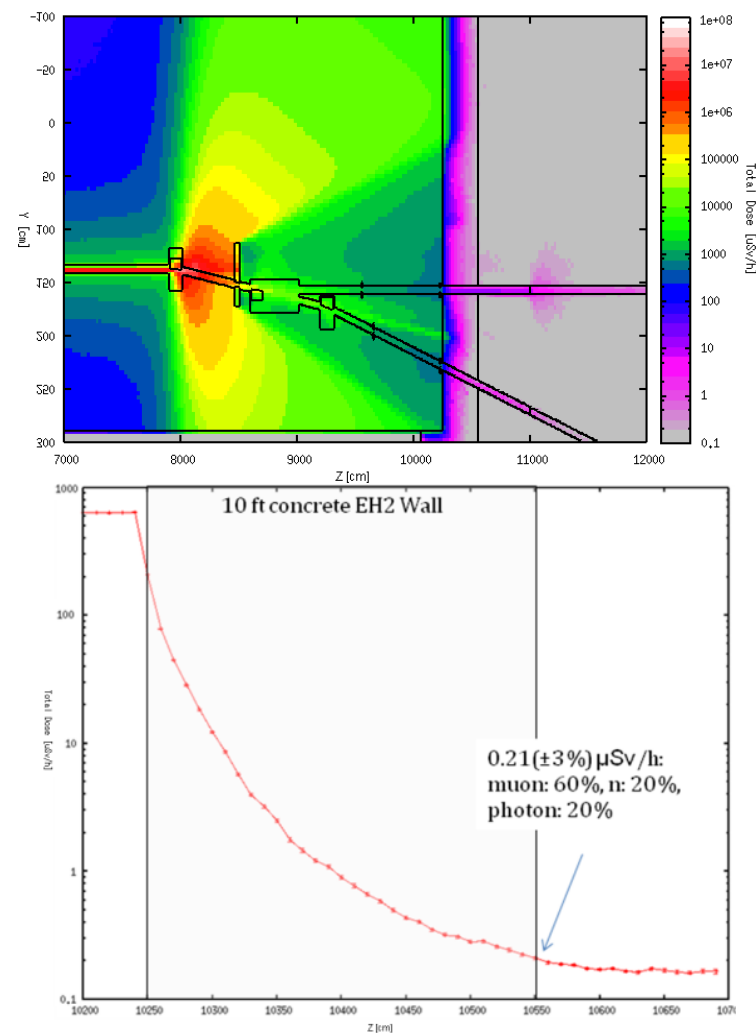

Figure 7. Total dose with a $40 \mathrm{~cm}$ iron safety collimator and $3 \mathrm{~m}$ concrete $\mathrm{EH} 2$ wall: $2 \mathrm{D}$ distribution on beam height and $1 \mathrm{D}$ on the primary incident direction.

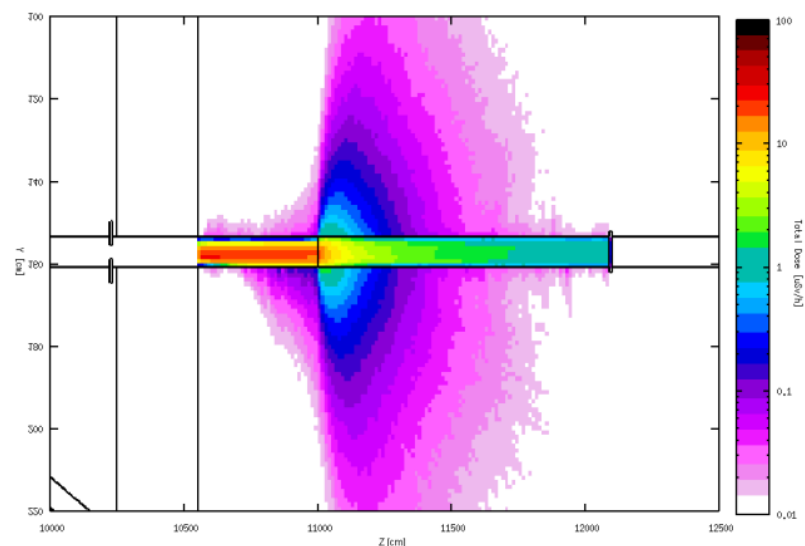

Figure 8. Total dose in $\mathrm{EH}^{2 k(k) \mid}$ due to radiation leakage from beam pipe.

\section{Conclusion}

Intensive Monte Carlo simulations have been performed for the shielding design for LCLS-II. The complicated geometry and beam loss scenarios in LCLS-II project require accurate simulation for shielding design. For the injector, a well designed wall can protect personnel during injector construction. Radiation safety aspects related to injector operation, such as penetration leakage and residual dose, are also addressed. For the XTOD, the application of local safety dump can help reduce the thickness and complexity of the main shielding wall separating LCLS-II tunnel and experimental hall, and that a proper collimator system can sufficiently limit radiation leakage through photon beam pipes.

\section{Acknowledgements}

This work was supported by Department of Energy contract DE-AC02-76-SFO0515.

\section{References}

[1] M. Santana Leitner, J.C. Liu, S.X. Mao, L. Nicolas, S.H. Rokni, S. Xiao, Radiation protection aspects in the design of the Linac Coherent Light Source, $12^{\text {th }}$ Intl. Conf. on Radiation Shielding (ICRS-12), Nara, Japan, Sep. 2-7, 2012, submitted to Progress in Nucl. \& Technol., (2013).

[2] G. Battistoni, S. Muraro, P.R. Sala, F. Cerutti, A. Ferrari, S. Roesler, A. Fassò and J. Ranft, The FLUKA code: Description and benchmarking, Proc. of the Had. Shower Sim. Workshop 2006, Fermilab Sep. 6-8, 2006, M. Albrow, R. Raja eds., AIP Conference Proceeding 896, 31-49, (2007).

[3] A. Ferrari, P.R. Sala, A. Fassò, and J. Ranft, FLUKA: a multi-particle transport code, CERN2005-10, SLAC-R-773, (2005).

[4] V. Vlachoudis, FLAIR: A powerful but user friendly graphical interface, Proc. Int. Conf. on Mathematics, Computational Methods \& Reactor Physics (M\&C 2009), Saratoga Springs, NY, May 3-7, 2009, (2009).

[5] S. Rokni, et al., LCLS-II Preliminary Design Report: Chapter13 Radiological Considerations, SLAC National Accelerator Laboratory, Menlo Park, CA, (2012). 